Eur J Nucl Med Mol Imaging (2004) 31:926

DOI 10.1007/s00259-004-1515-Z

Published online: 18 March 2004

(C) Springer-Verlag 2004

\section{Requirements for clinical PET: the situation in Switzerland}

\author{
Dear Sir,
}

I would like to comment on the article by Bedford and Maisey referring to the availability and requirements for clinical PET in Europe [1]. While this may be a minor point, Tables 3 and 5 both state that there are no PET scanners in Switzerland, although Prof. Maisey is well aware of our work. This is somewhat surprising. To clarify matters:

1. There are currently two PET-CT and seven PET scanners in Switzerland, a somewhat indeterminate fraction of which are used for research purposes (around two).

2. The number of scans done for NSCLC in Switzerland in 2003 was 1,582; thus underuse of PET in NSCLC in Switzerland is slightly more than 1,000 , according to Table 3.

3. The suggestion that 2,500-2,600 patients can be scanned on a single PET scanner (Table 3, second column, row Switzerland) may be correct in principle, but in practice I would be interested to know who in Europe is able to examine this many patients on a single scanner. Note that we did 2,100 PET-CT scans on a single PET-CT scanner in 2003, which is already a substantial load. It should not be forgotten that the number of scans done on a PET scanner is very much dependent on the type of scanner, the scanning protocol and, probably above all, the availability of FDG.

Gustav von Schulthess (

Division of Nuclear Medicine, Department of Medical Radiology, University Hospital, Rämistrasse 100, 8091 Zurich, Switzerland

e-mail: Gustav.vonschulthess@dmr.usz.ch

\section{Reference}

1. Bedford M, Maisey MN. Requirements for clinical PET: comparisons within Europe. Eur $\mathrm{J}$ Nucl Med Mol Imaging 2004;31:208-221
Eur J Nucl Med Mol Imaging (2004) 31:926-927

DOI 10.1007/s00259-004-1513-1

Published online: 18 March 2004

(C) Springer-Verlag 2004

\section{Requirements for clinical PET: clarification of the situation in Sweden}

Dear Sir,

We read with great interest the paper by Bedford and Maisey in which a method for assessing the requirements for introduction of positron emission tomography (PET) as a cancer imaging service was presented [1]. Studies like these, which attempt to objectify the actual need for a medical service, are important. We understand that simplifications had to be made in the study, and that the authors had to rely on the information most readily obtainable from different national sources.

Nevertheless, basic facts need to be as correct as possible, and we observe that neither the cited number of dedicated PET cameras nor information on their use for clinical examinations in Sweden is correct. The number of dedicated (human) PET scanners is stated to be seven, while there have never been more than a maximum of five in our country. One of them is an old and inefficient camera with limited usefulness, and this can hardly be included in the current survey. Consequently, there are in reality only four dedicated PET cameras in use in Sweden.

Furthermore, while Sweden has had a very active and long-standing tradition in PET (in Uppsala and Stockholm), this tradition has to a very large degree been directed towards basic research and methodological developments. It is only during the past few years that clinical PET in Sweden has achieved a "breakthrough" comparable to that happening internationally. Consequently, a correction regarding the number of cameras, similar to that made for the UK (Table 4 in the paper), is also necessary for Sweden. It is stated that in the UK there are 13 dedicated scanners, of which seven are in clinical use and six are research instruments. In Sweden, two dedicated PET cameras are effectively used for clinical purposes. This moves us from the authors' calculated position of number 2 in Europe (with 196.07\% of the number of scanners required), to sixth position, with only $56.02 \%$ available. After this adjustment, we lack two scanners instead of having an excess of three, according to the authors' model of estimated needs!

We would like to emphasise that we do not intend to criticise the authors' work or the method they present. We acknowledge that it is very difficult to analyse the imaging situation in accurate detail in all 36 different countries included. However, we do need to correct the unfortunately misleading information regarding the number of dedicated PET cameras currently available 